\title{
Disturbance observer-based controller for inverted pendulum with uncertainties: Linear matrix inequality approach
}

\author{
Van-Phong Vu, Minh-Tam Nguyen, Anh-Vu Nguyen, Vi-Do Tran, Tran-Minh-Nguyet Nguyen \\ Department of Automatic Control, Ho Chi Minh City University of Technology and Education, Ho Chi Minh City, \\ Vietnam
}

\begin{abstract}
Article Info
Article history:

Received Sep 4, 2020

Revised May 19, 2021

Accepted May 31, 2021

Keywords:

Disturbance observer

Inverted pendulum

LMIs

Observer-based controller

Uncertainties

ABSTRACT

A new approach based on linear matrix inequality (LMI) technique for stabilizing the inverted pendulum is developed in this article. The unknown states are estimated as well as the system is stabilized simultaneously by employing the observer-based controller. In addition, the impacts of the uncertainties are taken into consideration in this paper. Unlike the previous studies, the uncertainties in this study are unnecessary to satisfy the bounded constraints. These uncertainties will be converted into the unknown input disturbances, and then a disturbance observer-based controller will be synthesized to estimate the information of the unknown states, eliminate completely the effects of the uncertainties, and stabilize inverted pendulum system. With the support of lyapunov methodology, the conditions for constructing the observer and controller under the framework of linear matrix inequalities (LMIs) are derived in main theorems. Finally, the simulations for system with and without uncertainties are exhibited to show the merit and effectiveness of the proposed methods.
\end{abstract}

This is an open access article under the CC BY-SA license.

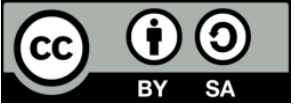

\section{Corresponding Author:}

Van-Phong $\mathrm{Vu}$

Department of Automatic Control

Ho Chi Minh City University of Technology and Education

No. 1 Vo Van Ngan Street, Thu Duc District, Ho Chi Minh city, Vietnam

Email: phongvv@hcmute.edu.vn

\section{INTRODUCTION}

Inverted pendulum system is a typical system which is used for developing and testing many modern control theories because of the interesting dynamic characteristics such as strong nonlinear, complicated, multi-variable, and unstable system. The model of inverted pendulum is quite similar to the practical models existing in reality such as a missile, self-balancing robot, and heavy crane lifting containers. In the past few decades, plenty of papers studying inverted pendulum have been published [1]-[12]. For example, the problems of modeling the inverted pendulum were investigated in papers [1] and [2] where the modeling method relied on the fuzzy cluster method was studied in [1] and the D'Alembert's principle was employed to model inverted pendulum in [2]. Additionally, the controller synthesis to stabilize the system has been received great attention from researchers [3]-[12]. For instance, a PI-state feedback controller was designed to control the inverted pendulum system in [5], in which the proportional and integral gains were determined via the pole placement method whose input control signal was sampled and did not have continuity of time. Another modern controller, sliding mode control, has been also applied to stabilize the inverted pendulum in [11], [12] as well. Unfortunately, the disadvantage of the sliding-mode approach is that there exist the chattering phenomena which will impact the devices and performance of the system. 
In reality, a lot of physical state variables of the system are unable or hard to measure by using the sensors. Moreover, employing sensors to obtain the information of the state variables will cause the cost to grow up and the sensors are also sensitive to the noise that leads to the incorrect measurements. Due to the above reasons, designing an observer to replace the sensors is a pressing issue that attracts a lot of researchers. Recently, there are many papers focusing on observer design [13]-[16].

Regarding observer design for inverted pendulum, several interesting results have been founded in some papers [17]-[19]. For example, an approach to design a high-order sliding mode observer was introduced in [17] to compute the unmeasurable states. However, the drawback of the sliding mode method in paper [17] is that the existence of the chattering phenomenon will influence the performance of the observer (to be seen in [17]). In paper [18], both states and faults were estimated by designing an observer and a method based on Ackerman's formula was presented in the article [18]. In the past decade, a mathematical technique called linear matrix inequality (LMI) which assists to solve the problems of the control field more easily and efficiently was introduced in [20]. However, to the best of our knowledge, there exist a few papers employing the LMI technique to synthesize observer for inverted pendulum. Thus, in this work, we will propose a method based on the LMI technique to construct an observer for inverted pendulum that can avoid the chattering issues in paper [17]. In addition, with the aid of the LMIs technique, the conditions to design observer in this article will be more relaxed with respect to the method employing ackerman's formula in paper [18].

Besides, in practice, the systems are inevitable to be impacted by the uncertainties which may originate from the modeling and/or parameter errors. The inverted pendulum is not an exception, hence, stabilizing the inverted pendulum with the impacts of the uncertainties is a pressing and interesting issue. There are many articles paying attention to stabilizing the uncertain inverted pendulum system in recent years [21]-[27]. In paper [21], a fuzzy type-2 PID controller was synthesized for the inverted pendulum to reject the influence of uncertainties and stabilize the inverted pendulum system. However, the uncertainties in paper [21] must be satisfied with the bounded constraints. An output feedback controller was proposed in paper [22] where the unknown states were estimated by the high-gain observer. However, there are several drawbacks in this work such as the high-gain observer is sensitive with measurement noises or sometimes the peaking phenomenon occurs due to the high gain of the observer. An adaptive controller and adaptive fuzzy sliding mode controller were synthesized for inverted pendulum and rotary inverted pendulum system with the uncertainties in [23], [24], respectively. A new approach based on the control lyapunov function and LMIs was investigated to synthesize the controller for inverted pendulum system with the existence of the uncertainties [25]. Regarding synthesizing for the uncertain inverted pendulum system, a sliding mode technique was employed to design an observer to calculate the unknown states and reject the impacts of the uncertainties. However, it is seen that the uncertainties of the previous articles [21]-[27] have to be bounded. It means that the upper and lower bounds of the uncertainties should be provided in advance, otherwise it is impossible to design the controller and observer for these systems.

Recently, a disturbance observer has been introduced to estimate the disturbance in [28]. This disturbance observer allows us to obtain information of the disturbance that needs to control the system and enhance the control accuracy of the system. There have been many previous papers studying about the disturbance observer such as papers [29]-[32]. Unfortunately, to the best of our knowledge, the disturbance observer has not been employed to deal with the inverted pendulum with the presence of the uncertainties in previous papers. Owing to this reason, we proposed a new approach relied on disturbance observer to estimate the unknown states and the uncertainties.

With the aforementioned analyses, it motivates us to propose a new method to synthesize the observer and disturbance observer-based controller for inverted pendulum emphasizing in the following contributions:

- An observer-based controller is synthesized to stabilize the inverted pendulum. The proposed method relying on the LMI technique allows us can determine the observer and controller gains easily and efficiently. The method in this paper will help to avoid the chattering phenomenon in paper [16] as well as the conditions for designing observer-based controller is also more relaxed in comparison with the method in [17]. In addition, some state variables of inverted pendulum are not measured by sensors, thus, the methods in [3]-[12] are failed to stabilize the system. To deal with these issues, in this article, an observer is synthesized for replacing the sensors to estimate the unknown states of the system.

- A disturbance observer-based controller is proposed for the inverted pendulum system with uncertainties that has not been found in any previous paper. Unlike previous papers [21]-[27], the uncertainties in this study do not need to fulfill the bounded constraints. Therefore, it is impossible to employ the methods in paper [21]-[27] to design a controller for our case. In this paper, first step, the uncertainties are transformed to the input disturbances, and then the disturbance observer-based controller is synthesized in the second step to estimate unknown states, and input disturbances simultaneously. This method has the 
advantages that the information of the uncertainties is obtained by observer, and then it is feed-backed to the controller to eliminate completely the impacts of the uncertainties and increase the control accuracy.

The rest of this article is organized as follows. In section 2, the research method that includes: the mathematical model of inverted pendulum is described, the problems will be solved, and methods to synthesize an observer-based controller based on LMI technique for inverted pendulum system without uncertainties in this paper are stated as well. The simulation results and discussions for both with and without uncertainties of inverted pendulum system are presented in section 3. Finally, several conclusions are summarized in section 4 .

Notations: In this paper, $\Theta>0(<0)$ indicates the matrix $\Theta$ is a positive (negative) definite. $\Theta^{T}$ represent the transpose of a matrix $\Theta ; \Theta^{-1}$ defined the inverse of $\Theta ; I$ is defined as an identity matrix. $\Theta^{+}$ indidcates the Moore-Penrose pseudo-inverse of $\Theta$ with $\Theta^{+}=\left(\Theta^{T} A\right)^{-1} \Theta^{T}$. The symbol $\mathfrak{R}^{n \times m}$ denotes the set of $n \times m$ matrices.

\section{RESEARCH METHOD}

\subsection{System model}

Consider the inverted pendulum on a cart in Figure 1 with the nonlinear equation as in (1):

$$
\left\{\begin{array}{l}
\ddot{\bar{x}}=\frac{u+m l \sin (\theta) \dot{\theta}^{2}-m g \cos (\theta) \sin (\theta)}{M+m-m(\cos (\theta))^{2}} \\
\ddot{\theta}=\frac{u \cos (\theta)-(M+m) g(\sin (\theta))+m l(\cos (\theta) \sin (\theta)) \dot{\theta}}{m l(\cos (\theta))^{2}-(M+m) l}
\end{array}\right.
$$

where the parameters and their values are described in Table 1 [33].

Let us define $x_{1}=\bar{x}, x_{2}=\dot{\bar{x}}, x_{3}=\theta, x_{4}=\dot{\theta}$, then the (1) is written as (2).

$$
\left[\begin{array}{c}
\dot{x}_{1} \\
\dot{x}_{2} \\
\dot{x}_{3} \\
\dot{x}_{4}
\end{array}\right]=\left[\begin{array}{c}
x_{2} \\
\frac{u \cos \left(x_{3}\right)-(M+m) g\left(\sin \left(x_{3}\right)+m l\left(\cos \left(x_{3}\right) \sin \left(x_{3}\right)\right) x_{4}\right.}{m l\left(\cos \left(x_{1}\right)\right)^{2}-(M+m) l} \\
x_{4} \\
\frac{u+m l\left(\sin \left(x_{3}\right)\right) x_{4}^{2}-m g \cos \left(x_{3}\right) \sin \left(x_{3}\right)}{M+m-m\left(\cos \left(x_{3}\right)\right)^{2}}
\end{array}\right]
$$

Linearizing the original nonlinear system (2) at the equilibrium point $(0,0,0,0)$ and subtitling the values of the parameters in Table 1 obtains the following system.

$$
\left\{\begin{array}{l}
\dot{x}(t)=A x(t)+B u(t) \\
y(t)=C x(t)
\end{array}\right.
$$

Where $=\left[\begin{array}{l}x_{1} \\ x_{2} \\ x_{3} \\ x_{4}\end{array}\right], \dot{x}=\left[\begin{array}{l}\dot{x}_{1} \\ \dot{x}_{2} \\ \dot{x}_{3} \\ \dot{x}_{4}\end{array}\right], A=\left[\begin{array}{cccc}0 & 1.00 & 0 & 0 \\ 0 & 0 & -1.9613 & 0 \\ 0 & 0 & 0 & 1 \\ 0 & 0 & 23.536 & 0\end{array}\right], B=\left[\begin{array}{c}0 \\ 1 \\ 0 \\ -2\end{array}\right]$, and $C=\left[\begin{array}{cccc}1 & 0 & 0 & 0 \\ 0 & 0 & 0 & 1\end{array}\right]$

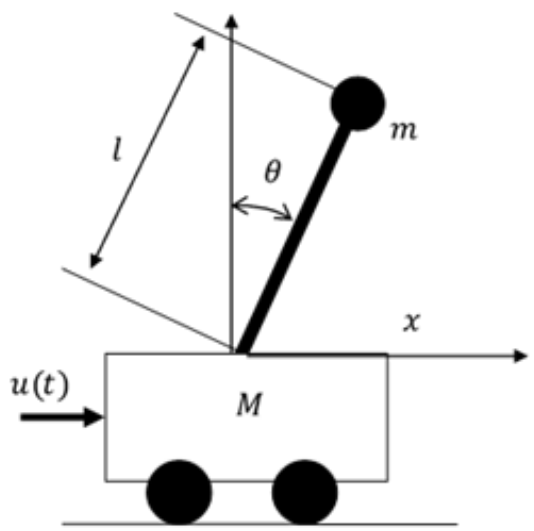

Figure 1. Inverted pendulum on car 
Table 1. Parameters of the inverted pendulum [33]

\begin{tabular}{cccc}
\hline Parameters & Symbol & Value & Unit \\
\hline Mass of cart & $\mathrm{M}$ & 1 & $\mathrm{~kg}$ \\
Mass of pendulum & $\mathrm{m}$ & 0.2 & $\mathrm{~kg}$ \\
Length of pole & 1 & 0.5 & $\mathrm{~m}$ \\
Gravitational acceleration & $\mathrm{g}$ & 9.80556 & $\mathrm{~m} / \mathrm{s}^{2}$ \\
Position of Cart & $\bar{x}$ & & $\mathrm{~m}$ \\
Velocity of Cart & $\dot{\bar{x}}$ & & $\mathrm{~m} / \mathrm{s}$ \\
Angle of inverted pendulum & $\theta$ & & $\mathrm{rad}$ \\
Angle velocity & $\dot{\theta}$ & & $\mathrm{rad} / \mathrm{s}$ \\
\hline
\end{tabular}

\subsection{Problem description}

Suppose that only the position of the cart $\left(x_{1}=x\right)$ and angle acceleration $\left(x_{4}=\dot{\theta}\right)$ are measured by sensors; and the velocity of the cart $\left(x_{2}=\dot{x}\right)$ and angle of inverted pendulum $\left(x_{3}=\theta\right)$ are unknown. However, the information of these two state variables is necessary to synthesize a controller to stabilize the system (3). Due to this reason, the objective of this paper is to design an observer-based controller to estimate the unknown state and stabilize the system (3) at the equilibrium point $(0,0,0,0)$. There are two scenarios taken into consideration in this article.

Scenario 1: The observer-based controller is synthesized for the inverted pendulum system (3) which is not affected by the uncertainties.

Scenario 2: The disturbance observer-based controller is designed for the inverted pendulum system which is affected by the uncertainties. It should be noted that the uncertainty in this case is unnecessary to satisfy the bounded constraints. In this case, both the unknown states and uncertainties are estimated asymptotically and feed-backed to the controller to stabilize the system.

Remark 1: In this paper, we assume that the velocity of the cart $\left(x_{2}=\dot{x}\right)$ and angle of inverted pendulum $\left(x_{3}=\theta\right)$ are not measured by sensors. It means that the information of these two state variables is unknown, therefore, the methods to design controller for inverted pendulum in papers [3]-[12] are unable to apply for this case. Additionally, in this study, sensors are not used for obtaining the information of velocity of the cart and angle of inverted pendulum leading to reduce the cost for constructing the system.

\subsection{Observer -based controller for inverted pendulum}

In this section, an observer and controller are designed for the system (3) simultaneously. The structure of the system with the observer-based controller is depicted in Figure 2. Let us take consideration the observer form for the system (3) as (4).

$$
\left\{\begin{array}{c}
\dot{\hat{x}}=A \hat{x}+B u+T(y-\hat{y}) \\
\hat{y}=C \hat{x}
\end{array}\right.
$$

Where $\hat{x}$ and $\hat{y}$ are the estimation of the state $x$ and output $y$, respectively. $T$ is the observer gain which is computed in next section. The controller form of the system (3) is expressed as (5).

$$
u=-K \hat{x}
$$

The estimation error is defined:

$$
e=x-\hat{x}
$$

The dynamic expression of the estimation error is:

$$
\dot{e}=\dot{x}-\dot{\hat{x}}
$$

Combining (3) and (4), one obtains:

$$
\dot{e}=(A-T C) e
$$

Substituting (5) into (3), the closed-loop system is obtained

$$
\dot{x}=(A-B K) x+B K e
$$


From (8) and (9), it infers that

$$
\begin{aligned}
& {\left[\begin{array}{l}
\dot{x} \\
\dot{e}
\end{array}\right]=\left[\begin{array}{cc}
A-B K & B K \\
0 & A-T C
\end{array}\right]\left[\begin{array}{l}
x \\
e
\end{array}\right]} \\
& \text { Denote } \tilde{x}=\left[\begin{array}{l}
x \\
e
\end{array}\right] \text { and } \tilde{A}=\left[\begin{array}{cc}
A-B K & B K \\
0 & A-T C
\end{array}\right], \text { then (10) becomes } \dot{\tilde{x}}=\tilde{A} \tilde{x}
\end{aligned}
$$

Theorem 1: The estimation error $e$ and the state variable $x$ of the system (3) with the observer (4) and controller (5) converge to zero asymptotically, if there exist matrices $T, K$, and positive symmetric matrices $P_{1}$ and $P_{2}$ such that the following condition holds

$$
\left[\begin{array}{cc}
(A-B K)^{T} P_{1}^{-1}+P_{1}^{-1}(A-B K) & P_{1}^{-1}(A-B K) \\
(B K)^{T} P_{1}^{-1} & (A-T C)^{T} P_{2}+P_{2}(A-T C)
\end{array}\right]<0
$$

Proof: The Lyapunov function is chosen as (13);

$$
V(t)=\tilde{x}^{T}(t) P \tilde{x}(t)
$$

where $P=\left[\begin{array}{cc}P_{1}^{-1} & 0 \\ 0 & P_{2}\end{array}\right]$

Taking the derivative on both sides of (13) yields;

$$
\dot{V}(t)=\dot{\tilde{x}}^{T}(t) P \tilde{x}(t)+\tilde{x}^{T}(t) P \dot{\tilde{x}}(t)
$$

From (11) and (14), we obtain;

$$
\begin{aligned}
& \dot{V}(t)=\tilde{x}^{T}(t)\left[\begin{array}{cc}
\tilde{A}^{T} P & P \tilde{A}] \tilde{x}(t)= \\
\tilde{x}^{T}(t)\left[\begin{array}{cc}
(A-B K)^{T} P_{1}^{-1}+P_{1}^{-1}(A-B K) & P_{1}^{-1}(A-B K) \\
(B K)^{T} P_{1}^{-1} & (A-T C)^{T} P_{2}+P_{2}(A-T C)
\end{array}\right] \tilde{x}(t)
\end{array}\right.
\end{aligned}
$$

It is easily seen that if the condition (12) is satisfied then $\dot{V}(t)<0$, it infers that $x$ and $e$ converge to zero asymptotically when $t \rightarrow \infty$. The proof is completed.

Unfortunately, it is obvious that there exist two matrix variables multiplying together in one term of the conditions (12), therefore condition (12) is a non-convex bilinear matrix inequality (BMI) that is complicated to determine matrix variables $T, K, P_{1}$, and $P_{2}$ fulfill the condition (12). Because of this reason, Theorem 2 needs to transform condition (12) to linear matrix inequality (LMI) which is easy to resolve by using the LMI tool of MATLAB.

Theorem 2: The estimation error $e$ and the state variable $x$ of the system (3) with the observer (4) and controller (5) approach zero asymptotically, if there exist matrices $T, K$, and positive symmetric matrices $P_{1}$ and $P_{2}$ such that the following conditions satisfy.

$$
\begin{aligned}
& P_{1} A^{T}-Z^{T} B^{T}+A P_{1}-B Z<0 \\
& A^{T} P_{2}-C^{T} W^{T}+P_{2} A-W C<0
\end{aligned}
$$

The observer and controller gains obtain

$$
\begin{aligned}
& T=P_{2}^{-1} W \\
& K=Z P_{1}^{-1}
\end{aligned}
$$

Proof: According to the Schur complement, (12) is equivalent to

$$
\left\{\begin{array}{c}
(A-B K)^{T} P_{1}^{-1}+P_{1}^{-1}(A-B K)<0(20 a) \\
(A-T C)^{T} P_{2}+P_{2}(A-T C)<0(20 b)
\end{array}\right.
$$

Pre and post multiplying $P_{1}$ with (20a) and denoting $Z=K P_{1}$, (20a) becomes 
$P_{1} A^{T}-Z^{T} B^{T}+A P_{1}-B Z<0$

Define $W=P_{2} T$ then (20b) is written as (22),

$$
A^{T} P_{2}-C^{T} W^{T}+P_{2} A-W C<0
$$

From (21) and (22), it is obvious that (21) and (22) are the LMIs and they are the same as (16) and (17) of Theorem 2. It means that it is successful to convert BMI (12) of Theorem 1 to LMIs (16) and (17) of Theorem 2. The proof is completed.

The procedure for synthesizing is briefly presented as follows.

Step 1: Solving the LMI (16) and (17) to obtain matrices $\mathrm{Z}, \mathrm{P}_{1}, \mathrm{~W}$, and $\mathrm{P}_{2}$.

Step 2: The observer and controller gains $T$ and $K$ are computed by using (18) and (19).

\subsection{Disturbance observer -based controller for inverted pendulum with uncertainties}

Assume that the inverted pendulum system (1) is impacted by the time-varying uncertainties, then it is rewritten in the following framework (23):

$$
\left\{\begin{array}{l}
\dot{x}(t)=(A+\Delta A(t)) x(t)+(B+\Delta B(t)) u(t) \\
y(t)=C x(t)
\end{array}\right.
$$

where $\Delta A(t)$ and $\Delta B(t)$ are the uncertainties.

With the existence of the uncertainties, the performance of the system (1) is degraded. Hence, the objective of this section is to synthesize an observer-based controller to eliminate the effects of the uncertainties and stabilize the system. Because of the existence of the uncertainties, the method to synthesize the observer-based controller in section 2.3 are failed to apply for the system (1). Therefore, in this section, a new method based on the disturbance-observer based controller is proposed to stabilize the system (1). The structure of the observer-based controller for system with uncertainties is shown in Figure 3.

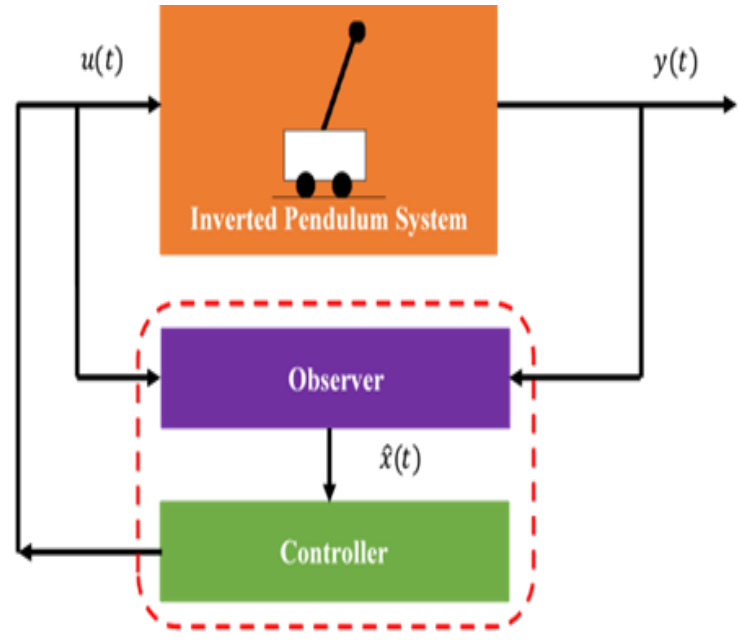

Figure 2. Structure of observer-based controller for inverted pendulum system

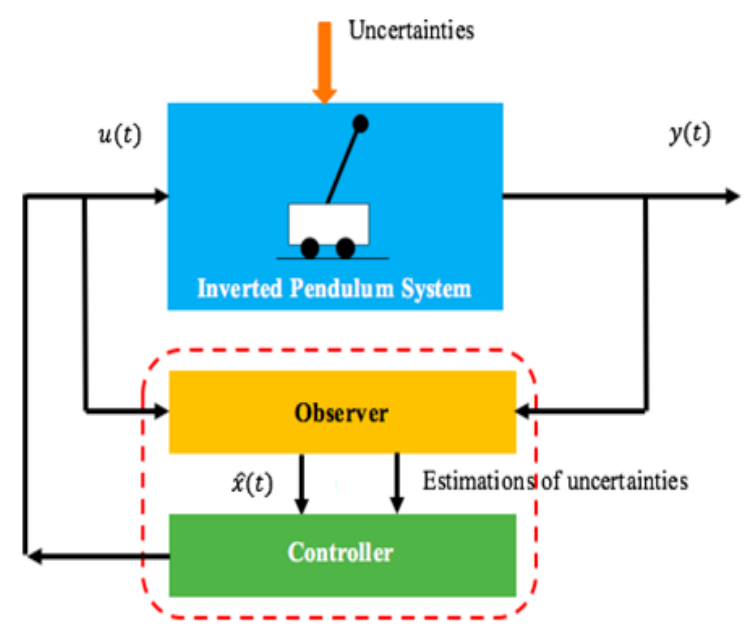

Figure 3. Structure of observer-based controller for inverted pendulum system with uncertainties

Assumption 1: The uncertainties $\Delta A(t)$ and $\Delta B(t))$ are supposed to satisfy the following matching conditions:

$$
\Delta A(t)=B \gamma(t) \text { and } \Delta B(t)=B \delta(t) .
$$

Remark 2: It should be noted that the uncertainties $\Delta A(t)$ and $\Delta B(t))$ in (23) do not need to satisfy the bounded constraints and the lower and upper-bounded value are unknown, or in practice, it is difficult to determine the values of the lower/upper bounds of the uncertainties. Therefore, it is impossible to apply the methods in papers [23-27] for synthesizing controller for the system in (23). Because the previous studies in papers [23-27], the upper bounds of uncertainties must be included in the conditions to design observer and 
controller. Owing to this reason, a new approach based on disturbance observer-based controller to eliminate the influences of the uncertainties and stabilize the system is investigated in this section.

Remark 3: The matching conditions in assumption 1 are needed to transform the uncertainties into the unknown input disturbance. This assumption can be found in previous papers such as [15], [16] and [34]. However, we assume that this assumption is still conservative when common matrix $B$ is used to decompose the uncertainties $\Delta A(t)$ and $\Delta B(t)$. It is still an open issue that need to solve in future work.

Lemma 1 [35]: Taken into account of the matrix equation $X A=B$ where $A \in \mathbb{R}^{m \times n}, m \geq n$, and $B \in \mathbb{R}^{k \times n}$. The general solution of the above matrix equation is expressed in the form $X=B A^{+}+Y\left(I-A A^{+}\right)$in which $Y \in \mathbb{R}^{k \times m}$ is an arbitrary matrix with appropriate dimension and $A^{+}=\left(A^{T} A\right)^{-1} A^{T}$ is the Moore-Penrose pseudoinverse of $A$.

Under assumption 1, the system (23) becomes (24):

$$
\left\{\begin{array}{l}
\dot{x}(t)=A x(t)+B u(t)+B(\gamma(t) x(t)+\delta(t) u(t)) \\
y(t)=C x(t)
\end{array}\right.
$$

Denote

$$
\omega(t)=\gamma(t) x(t)+\delta(t) u(t)
$$

Then the system (24) is modified as (25):

$$
\left\{\begin{array}{l}
\dot{x}(t)=A x(t)+B(u(t)+\omega(t)) \\
y(t)=C x(t)
\end{array}\right.
$$

It is seen that under assumption 1, the inverted pendulum system (23) with uncertainties has been transformed to the system (25) with the unknown input disturbance. From now on, the controller and observer will be synthesized for the system (25) instead of (23). Additionally, in this section, a disturbance observer is synthesized for the system (25) to estimate the unknown states and the disturbance $\omega(t)$ simultaneously. After that this information is feed-backed to the controller to make the Inverted Pendulum stable at the equivalent point $(0,0,0,0)$.

Consider the disturbance observer form as (26):

$$
\left\{\begin{array}{l}
\dot{\eta}(t)=X \eta(t)+T u(t)+J y(t) \\
\hat{x}(t)=\eta(t)-E y(t) \\
\widehat{\omega}(t)=(C B)^{+} \dot{y}(t)-S \hat{x}(t)-u(t)
\end{array}\right.
$$

in which $\hat{x}(t)$ and $\widehat{\omega}(t)$ are the estimation of the state $x(t)$, and disturbance $\omega(t)$, respectively. $X, T, J, E$, and $S$ are the observer gains of the observer (26). $(C B)^{+}=\left[(C B)^{T}(C B)\right]^{-1}(C B)$ is the Moore-Pseudo invert of $(C B)$.

The controller form is expressed in the following framework (27):

$$
u(t)=-K \hat{x}(t)-\widehat{\omega}(t)
$$

Let us define the estimation errors:

$$
e(t)=\hat{x}(t)-x(t)
$$

Substituting (26) into (28) yields:

$$
e(t)=\eta(t)-E C x(t)-x(t)=\eta(t)-M x(t)
$$

where $M=[I+E C]$

Taking the derivative of (29), one obtains:

$$
\dot{e}(t)=\dot{\eta}(t)-M \dot{x}(t)
$$

From (25), (26) and (30), we have: 
$\dot{e}(t)=[X \eta(t)+T u(t)+J y(t)]-M[A x(t)+B(u(t)+\omega(t))]$

$=X e(t)+[X M-M A+J C] x(t)+[T-M B] u(t)-M B \omega(t)$

Denote the estimation error of the disturbance:

$$
e_{\omega}(t)=\widehat{\omega}(t)-\omega(t)
$$

Combining (23), (26), and (32) obtains (33):

$$
\begin{aligned}
& e_{\omega}(t)=(C B)^{+} C[A x+B(u+\omega(t))]-S \hat{x}(t)-u(t)-\omega(t) \\
& \left.=(C B)^{+} C A x(t)+(C B)^{+}(C B) u+(C B)^{+}(C B) \omega(t)\right)-S \hat{x}(t)-u(t)-\omega(t) \\
& =-S e(t)+\left[S-(C B)^{+} C A\right] x(t)
\end{aligned}
$$

Theorem 3: The states of the system (23), the estimation errors of states (28), and the estimation error of the disturbance (32) approach zero asymptotically if there exist the positive symmetric matrix $\bar{Q}$, and the matrices $K, X, T, J, E$, and $S$ such that the following conditions satisfy:

$$
\begin{aligned}
& X M-M A+J C=0 \\
& T-M B=0 \\
& M B=0 \\
& S-(C B)^{+} C A=0 \\
& \Xi^{T} \bar{Q} \tilde{x}(t)+\bar{Q} \Xi<0
\end{aligned}
$$

where $Q=\left[\begin{array}{cc}\varepsilon Q^{-1} & 0 \\ 0 & R\end{array}\right], \Xi=\left[\begin{array}{cc}A-B K & B K+S \\ 0 & S\end{array}\right], \varepsilon$ is a positive scalar.

Proof: If the conditions (34)-(37) hold then the dynamic estimation error (31) and the disturbance estimation error (33) becomes (39);

$$
\dot{e}(t)=X e(t)
$$

and

$$
e_{\omega}(t)=-S e(t)
$$

From (40), it is seen that if the estimation error $e(t) \rightarrow 0$ when $t \rightarrow \infty$ then the estimation error of the disturbance in (40) converges to zero as well. Therefore, we merely need to synthesize the observer to make the estimation error approach zero then the estimation error of the disturbance automatically converges to zero.

From (23), (27), and (40), it infers that

$$
\begin{aligned}
& \dot{x}(t)=A x(t)-B K \hat{x}(t)-\widehat{\omega}(t)+\omega(t) \\
& =(A-B K) x(t)+(B K-S) e(t)
\end{aligned}
$$

Combining (39) and (41) yields:

$$
\left[\begin{array}{c}
\dot{x}(t) \\
\dot{e}(t)
\end{array}\right]=\left[\begin{array}{cc}
A-B K & B K-S \\
0 & X
\end{array}\right]\left[\begin{array}{l}
x(t) \\
e(t)
\end{array}\right]
$$

Denote;

$$
\tilde{x}(t)=\left[\begin{array}{l}
x(t) \\
e(t)
\end{array}\right], \Xi=\left[\begin{array}{cc}
A-B K & B K-S \\
0 & X
\end{array}\right] \text { then (42) become } \dot{\tilde{x}}(t)=\Xi \tilde{x}(t)
$$

Choose the lyapunov function; 


$$
V(\tilde{x}(t))=\tilde{x}^{T}(t) \bar{Q} \tilde{x}(t)
$$

in which $\bar{Q}=\left[\begin{array}{cc}\varepsilon Q^{-1} & 0 \\ 0 & R\end{array}\right]$

From (44), we can have (45);

$$
\dot{V}(\tilde{x}(t))=\dot{\tilde{x}}^{T}(t) \bar{Q} \tilde{x}(t)+\tilde{x}^{T}(t) \bar{Q} \dot{\tilde{x}}(t)
$$

Combining (43) and (45) yields (46);

$$
\dot{V}(\tilde{x}(t))=[\Xi \tilde{x}(t)]^{T} \bar{Q} \tilde{x}(t)+\tilde{x}^{T}(t) \bar{Q}[\Xi \tilde{x}(t)]=\tilde{x}^{T}(t)\left[\Xi^{T} \bar{Q} \tilde{x}(t)+\bar{Q} \Xi\right] \tilde{x}(t)
$$

From (46), it is seen that if the condition (38) of Theorem 3 holds then $\dot{V}(\tilde{x}(t))<0$, it means that $\tilde{x}(t)=$ $\left[\begin{array}{l}x(t) \\ e(t)\end{array}\right] \rightarrow 0$ when $t \rightarrow \infty$ asymptotically. The proof is successfully completed.

Unfortunately, the condition (38) of Theorem 3 is BMI that is hard to resolve in MATLAB to obtain both observer and controller gains. The following Theorem is needed to transform the BMI (38) into LMI.

Theorem 4: The states of the system (23), the estimation error of the state variables (28), and the estimation error of the disturbance (32) converge to zero asymptotically if there exist the positive symmetric matric $Q$, $R$, and the matrices $K, X, T, J, E$, and $S$ such that the following conditions hold:

$$
\begin{aligned}
& A^{T} R+R A+A^{T} C^{T} \Gamma^{T} R+R \Gamma C A+A^{T} C^{T} \Omega^{T} \bar{Z}^{T}+\bar{Z} \Omega C A-C^{T} Y^{T}-Y C<0 \\
& Q A^{T}+A Q-W^{T} B^{T}-B W<0 \\
& \text { where } E=\Gamma+Z \Omega \\
& \Gamma=-B(C B)^{+} \\
& \Omega=I-(C B)(C B)^{+} \\
& Y=R L \\
& \bar{Z}=R Z \\
& W=K Q
\end{aligned}
$$

The observer and controller gains are computed as (55)-(59):

$$
\begin{aligned}
& X=M A-L C \\
& J=L(I+C E)-M A E \\
& T=M B \\
& S=(C B)^{+} C A \\
& K=W Q^{-1}
\end{aligned}
$$

Proof: From (38), one obtains (26);

$$
\begin{aligned}
& {\left[\begin{array}{cc}
A-B K & B K-S \\
0 & X
\end{array}\right]^{T}\left[\begin{array}{cc}
\varepsilon Q^{-1} & 0 \\
0 & R
\end{array}\right]+\left[\begin{array}{cc}
\varepsilon Q^{-1} & 0 \\
0 & R
\end{array}\right]\left[\begin{array}{cc}
A-B K & B K-S \\
0 & X
\end{array}\right]=} \\
& =\left[\begin{array}{cc}
\varepsilon\left[A^{T} Q^{-1}+Q^{-1} A-K^{T} B^{T} Q^{-1}-Q^{-1} B K\right] & \varepsilon\left[Q^{-1} B K-Q^{-1} S\right] \\
\varepsilon\left[K^{T} B^{T} Q^{-1}-S^{T} Q^{-1}\right] & X^{T} R+R X
\end{array}\right]<0
\end{aligned}
$$

Let us define $\Lambda=A^{T} Q^{-1}+Q^{-1} A-K^{T} B^{T} Q^{-1}-Q^{-1} B K, \Delta=Q^{-1} B K-Q^{-1} S$, and $\Phi=X^{T} R+$ $R X$, then substituting into (60) yields (61); 


$$
\left[\begin{array}{cc}
\varepsilon \Lambda & \varepsilon \Delta \\
(*) & \Phi
\end{array}\right]<0
$$

According to Schur complement, (61) is equivalent to (62);

$$
\left\{\begin{array}{c}
\Phi<0 \\
\varepsilon \Lambda-\varepsilon \Delta(\Phi)^{-1}(\varepsilon \Delta)^{T}<0
\end{array}\right.
$$

Because $\Phi<0, \varepsilon \Delta(\Phi)^{-1}(\varepsilon \Delta)^{T} \leq 0$. Thus, $\Lambda<\varepsilon \Delta(\Phi)^{-1}(\Delta)^{T} \leq 0$. Then (62) is equivalent to (63);

$$
\left\{\begin{array}{l}
\Phi<0 \\
\Lambda<0
\end{array}\right.
$$

in which $\Phi=X^{T} R+R X$ and $\Lambda=A^{T} Q^{-1}+Q^{-1} A-K^{T} B^{T} Q^{-1}-Q^{-1} B K$

Firstly, let us take into account of the matrix inequality (63), it infers that (64);

$$
\Phi=X^{T} R+R X<0
$$

It is easily seen that $R$ and $X$ are both variables, hence (64) is a BMI that is hard to solve in Matlab. The following steps are to convert BMI (64) to LMI. From (36), we have (65), (66);

$$
\begin{aligned}
& {[I+E C] B=0} \\
& E(C B)=-B
\end{aligned}
$$

According to Lemma 1, the general solution of (66) is (67):

$$
E=\Gamma+Z \Omega
$$

in which $\Gamma=-B(C B)^{+}, \quad \Omega=I-(C B)(C B)^{+}, Z$ is an arbitrary matrix with a compatible dimension. Adding a slack variable $L$ which is defined as (68).

$$
L=J+X E
$$

From (34) and (68), it yields (69);

$$
X=M A-L C
$$

Combining (68) and (69) yields (70);

$$
J=L(I+C E)-M A E
$$

Substituting (67) into (69) yields (71);

$$
X=(I+E C) A-L C=(I+(\Gamma+Z \Omega) C) A-L C
$$

Substituting (71) into (64) results in (72);

$$
\begin{aligned}
\Theta & =[(I+(\Gamma+Z \Omega) C) A-L C]^{T} R+R[(I+(\Gamma+Z \Omega) C) A-L C] \\
& =A^{T} R+R A+A^{T} C^{T} \Gamma^{T} R+R \Gamma C A+A^{T} C^{T} \Omega^{T} Z^{T} R+R Z \Omega C A-C^{T} L^{T} R-R L C<0
\end{aligned}
$$

Denote

$$
\begin{aligned}
& Y=R L \\
& \bar{Z}=R Z \\
& \bar{Z}=R Z
\end{aligned}
$$


Then substitute them into (72) and yields (75);

$$
\Theta=A^{T} R+R A+A^{T} C^{T} \Gamma^{T} R+R \Gamma C A+A^{T} C^{T} \Omega^{T} \bar{Z}^{T}+\bar{Z} \Omega C A-C^{T} Y^{T}-Y C<0
$$

The matrices $A, C, E$ are the known matrices; $R, \bar{Z}$, and $Y$ are the matrix variables, it is obvious that each term of the matrix inequality of (75) only contains one variable, therefore (75) is a LMI which can be solved in MATLAB. Similarly, the inequality (63) is a BMI as well, therefore, this BMI is transformed to LMI in the following steps. The inequality (63) infers that:

$$
\Sigma=A^{T} Q^{-1}+Q^{-1} A-K^{T} B^{T} Q^{-1}-Q^{-1} B K<0
$$

Pre and post multiplying $Q$ to (76) yields (77):

$$
\Sigma=Q A^{T}+A Q-Q K^{T} B^{T}-B K Q<0
$$

Define

$$
W=K Q
$$

From (77) and (78), we have (79),

$$
\Sigma=Q A^{T}+A Q-W^{T} B^{T}-B W<0
$$

It is seen that each term of the inequality (41) merely contain one variable, therefore (41) is an LMI. From (37) and (41), it is seen that the BMI (63) has been transformed to (80):

$$
\left\{\begin{array}{l}
\Theta<0 \\
\Sigma<0
\end{array}\right.
$$

where $\Theta=A^{T} R+R A+A^{T} C^{T} \Gamma^{T} R+R \Gamma C A+A^{T} C^{T} \Omega^{T} \bar{Z}^{T}+\bar{Z} \Omega C A-C^{T} Y^{T}-Y C$ and $\quad \Sigma=Q A^{T}+A Q-$ $W^{T} B^{T}-B W$

Therefore, the BMI (38) of Theorem 3 is successfully converted into LMI (47) and (48) of theorem 4. The proof is completed. For the sake of easy understanding, the procedure to determine the observer and controller gains is briefly summarized as follows:

Step 1: The matrices $\Gamma$ and $\Omega$ are obtained from (50) and (51), respectively.

Step 2: Solving the LMI (47) and (48), we get the matrices $Q, R, Y, \bar{Z}$, and $W$. Then, $E, L$, and $Z$ are calculated from (49), (52), and (53), respectively.

Step 3: The observer gains $X, T, J, E$, and $S$ are computed from (55)-(58) and the controller gains are determined from (59).

\section{RESULTS AND DISCUSSION}

In this section, we will design an observer-based controller for the inverted pendulum with the parameters in Table 1. The LMIs of the main theorems are solved by using LMI solver with feasp function to obtain the observer and controller gains.

Scenario 1: Observer-based controller design for an inverted pendulum system without uncertainties In this case, an observer-based controller will be synthesized for the inverted pendulum without uncertainties. Solving the conditions of theorem 2, the observer and controller gains are obtained observer gain: $L=\left[\begin{array}{rr}0.9294 & -0.5735 \\ 1.3098 & -2.1525 \\ 0.5735 & 0.9294 \\ 0.1912 & 24.8457\end{array}\right]$ and controller gain: $K=\left[\begin{array}{llll}-4.1540 & -5.2156 & -52.1325 & -7.1705\end{array}\right]$.

By using Simulink of MATLAB, the simulation results are obtained in Figures 4-7.

Discussion 1: By using MATLAB/Simulink to simulate the system, the simulation results are shown in Figures 4-7. From the simulation results in Figures 4-7, it is seen that the estimated states $\hat{x}_{1}(t), \hat{x}_{2}(t), \hat{x}_{3}(t)$, and $\hat{x}_{4}(t)$ approach to real states displacement $x_{1}(t)$, velocity $x_{2}(t)$, angle $x_{3}(t)$, and angle velocity $x_{4}(t)$ asymptotically. And all estimation errors $e_{1}(t), e_{2}(t), e_{3}(t)$, and $e_{4}(t)$ converge to zero. Moreover, all states $x_{1}(t)$, velocity $x_{2}(t)$, angle $x_{3}(t)$, and angle velocity $x_{4}(t)$ quickly approach to zero after 10 seconds. It proves that the proposed method is successful to design the observer-based controller for stabilizing the 
inverted pendulum system without the influence of uncertainties. The observer-based controller not only estimates the unknown states but also control the inverted pendulum stably at equilibrium point $(0,0,0,0)$.

Scenario 2: Disturbance observer-based controller synthesis for an inverted pendulum system with uncertainties.

Assume that the inverted pendulum system is impacted by the time-varying uncertainties $\Delta A(t)$ and $\Delta B(t)$. Under Assumption 1, these uncertainties are decomposed as $\Delta A(t)=B \gamma(t)$ and $\Delta B(t)=B \delta(t)$ in

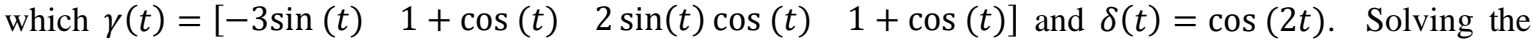
conditions of Theorem 4 , we obtain the observer and controller gains as:
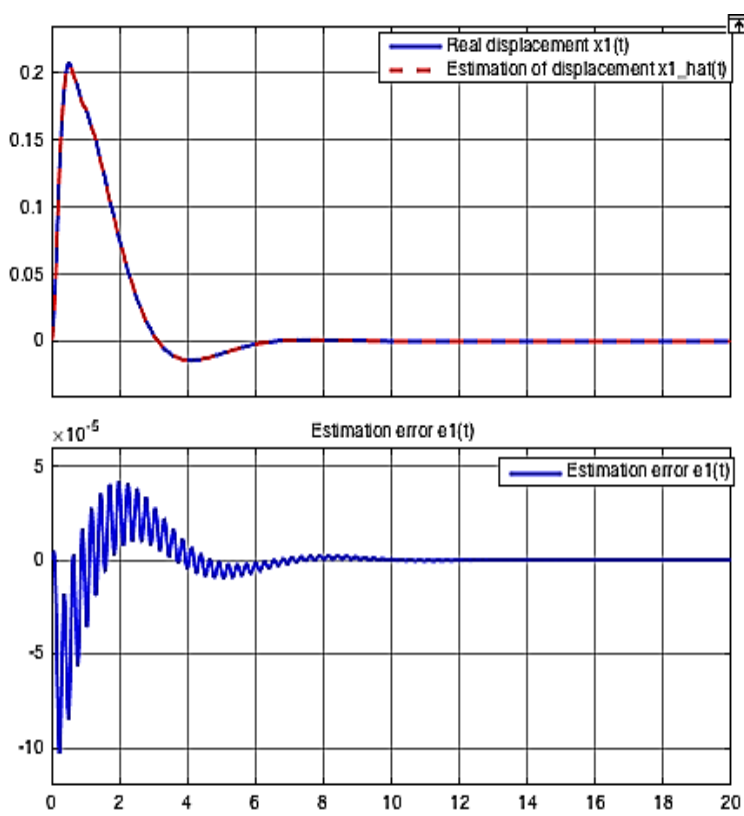

Figure 4. The real displacement $x_{1}(t)$, estimated displacement $\hat{x}_{1}(t)$ and estimation error $e_{1}(t)$
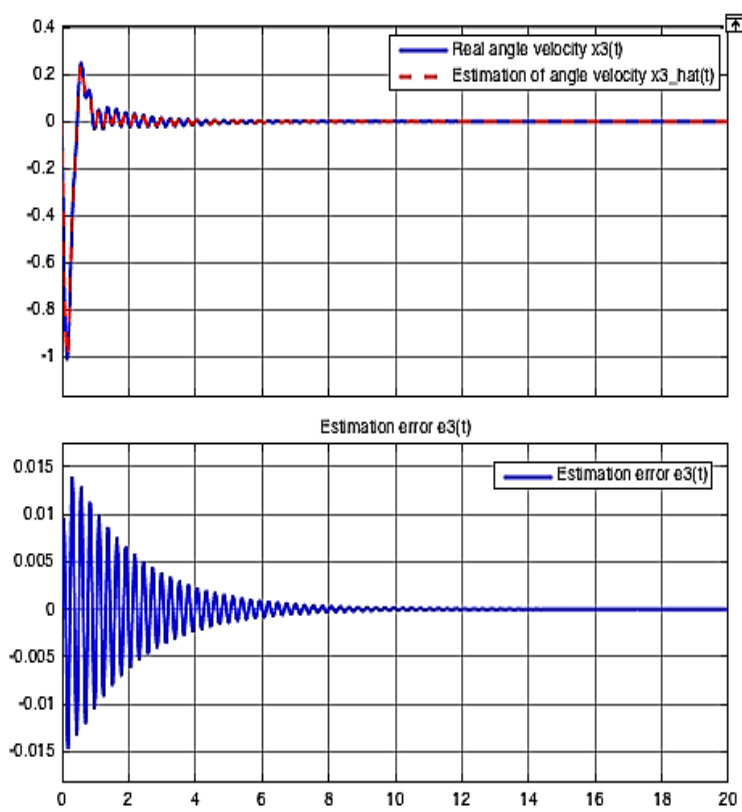

Figure 6 . The real angle $x_{3}(t)$, estimated angle $\hat{x}_{3}(t)$ and estimation error $e_{3}(t)$
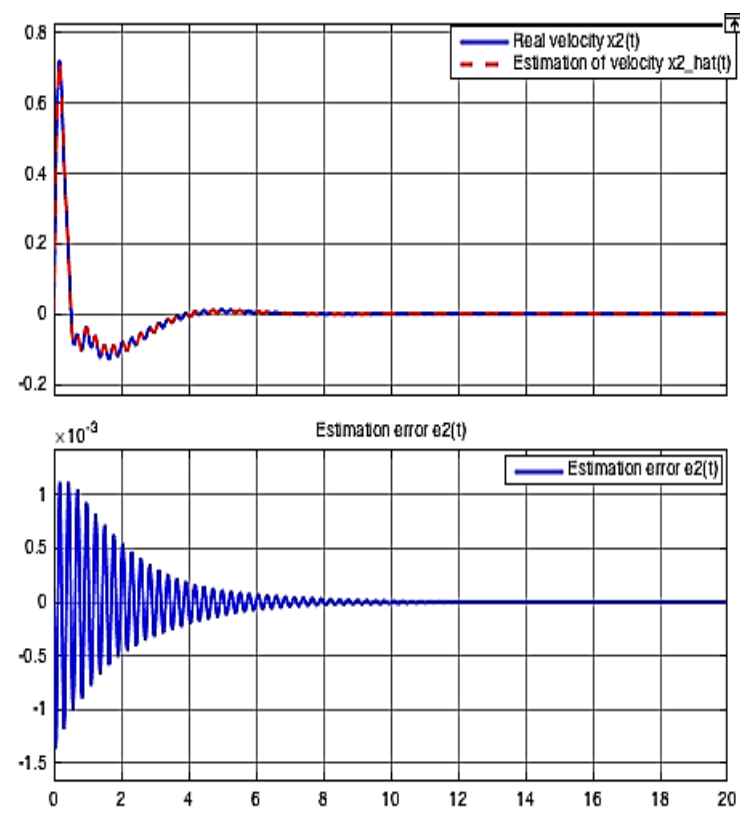

Figure 5 . The real velocity $x_{2}(t)$, estimated velocity $\hat{x}_{2}(t)$ and estimation error $e_{2}(t)$
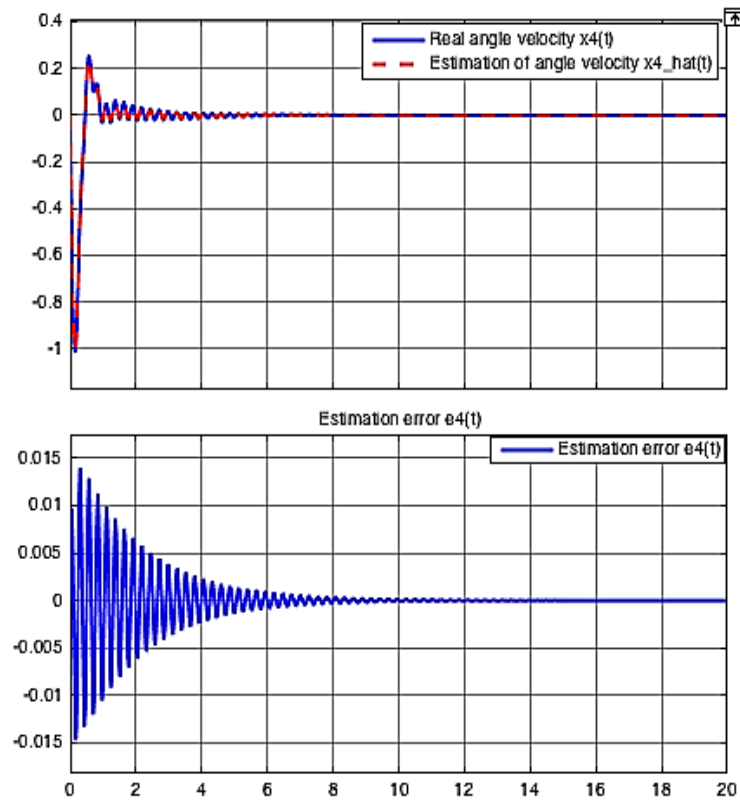

Figure 7. The real angle velocity $x_{4}(t)$, estimated angle velocity $\hat{x}_{4}(t)$ and estimation error $e_{4}(t)$ 


$$
\begin{aligned}
X & =\left[\begin{array}{cccc}
-0.5 & -1.9238 & 0 & -0.00 \\
1.9420 & -1.0069 & 9.8067 & -0.2511 \\
0.0992 & -9.8581 & 0 & -0.0128 \\
0.000 & 0.2488 & 0 & -0.5000
\end{array}\right], J=\left[\begin{array}{ccc}
-2.8989 & 0.9619 \\
99.4603 & 0.5034 \\
-9.7320 & 5.9291 \\
0.3749 & -0.1244
\end{array}\right], \\
T & =\left[\begin{array}{l}
0 \\
0 \\
0 \\
0
\end{array}\right], E=\left[\begin{array}{ccc}
-2.9238 & 0 \\
-1.0069 & 0.5000 \\
-9.8581 & 0 \\
0.2488 & -1.0000
\end{array}\right] \\
S & =\left[\begin{array}{llll}
0 & 0 & -11.7680 & 0
\end{array}\right] ; K=\left[\begin{array}{llll}
-5.3037 & -6.0658 & -55.1568 & -8.2875
\end{array}\right] .
\end{aligned}
$$

After carrying out simulating the system by simulink tool, the results are shown in Figures 8-11.
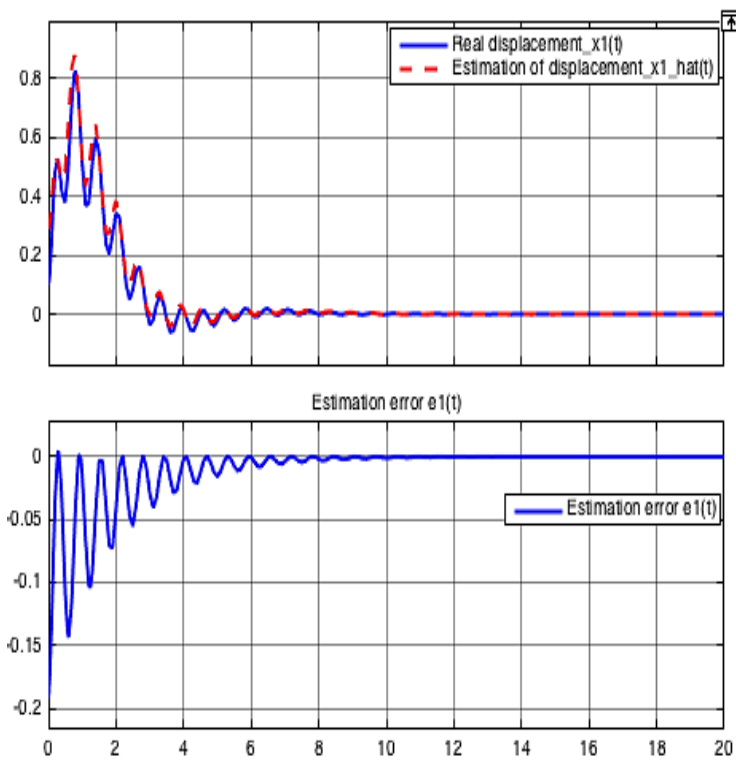

Figure 8. The real displacement $x_{1}(t)$, estimated displacement $\hat{x}_{1}(t)$ and estimation error $e_{1}(t)$
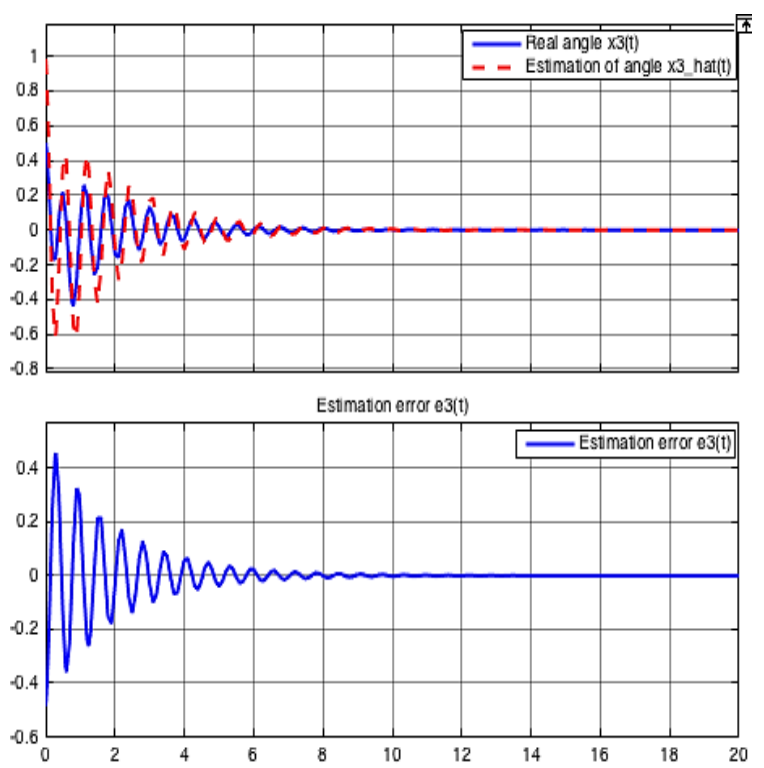

Figure 10. The real angle $x_{3}(t)$, estimated angle $\hat{x}_{3}(t)$ and estimation error $e_{3}(t)$

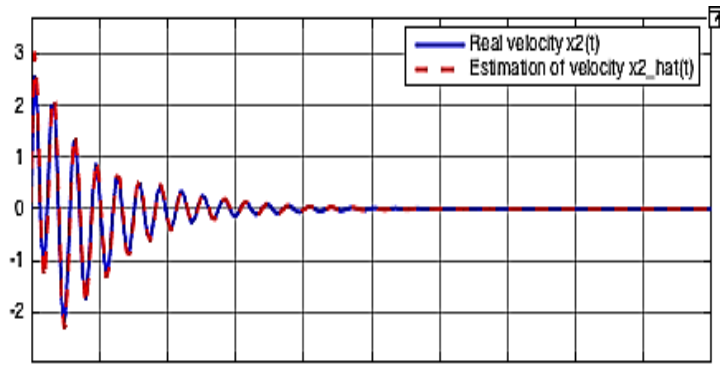

Estimation error e2(t)

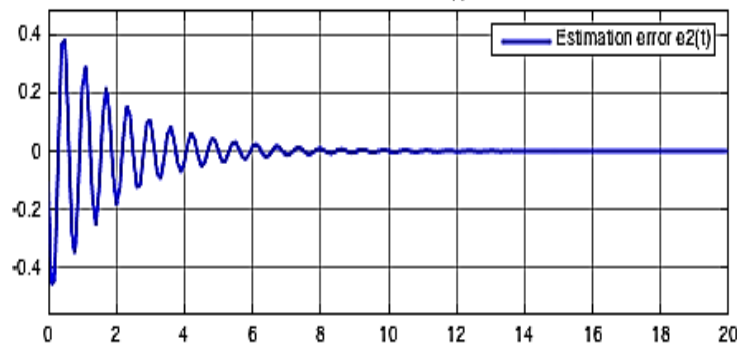

Figure 9 . The real velocity $x_{2}(t)$, estimated velocity $\hat{x}_{2}(t)$ and estimation error $e_{2}(t)$
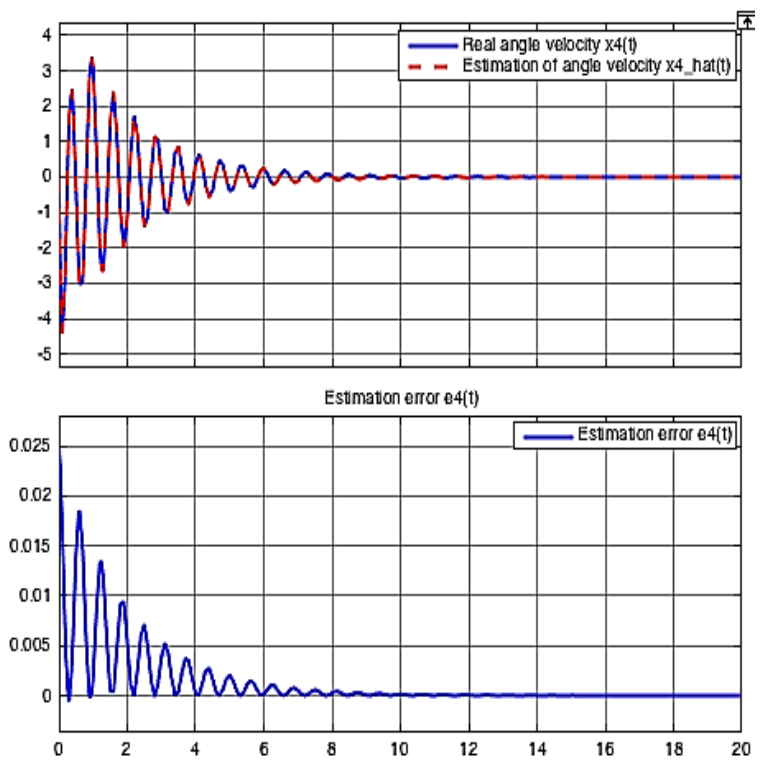

Figure 11 . The real angle velocity $x_{4}(t)$, estimated angle velocity $\hat{x}_{4}(t)$ and estimation error $e_{4}(t)$ 
Discussion 2: In this case, it is assumed that inverted pendulum system is impacted by the uncertainties. The uncertainties will influence the performance of the system. However, with the simulation results shown in Figures 8-11, it is obvious that the observer still operates very well, the real states of displacement $x_{1}(t)$, velocity $x_{2}(t)$, angle $x_{3}(t)$, and angle velocity $x_{4}(t)$ are estimated successfully and all estimation errors $e_{1}(t), e_{2}(t), e_{3}(t)$, and $e_{4}(t)$ approach zero asymptotically after shot time (less than 10 seconds). Furthermore, all states $x_{1}(t)$, velocity $x_{2}(t)$, angle $x_{3}(t)$, and angle velocity $x_{4}(t)$ of inverted pendulum system also have fast-convergence to equilibrium point (around 10 seconds). Thus, we can conclude that our method is successful to synthesize the disturbance observer-based to reject the influences of uncertainties, estimate unknown states and stabilize the inverted pendulum system with uncertainties.

\section{CONCLUSION}

In this paper, the observer-based controller and a disturbance observer-based controller have been designed for the inverted pendulum system without and with uncertainties, respectively. The observer is synthesized to estimate both the information of uncertainties and unknown states simultaneously and the controller is designed for eliminating the impacts of uncertainties and stabilizing system. The conditions expressed in term of LMIs framework for designing observer-based controller are derived in four main Theorems. Finally, the simulation results have illustrated that the proposed method is successful to stabilize the inverted pendulum system with/without uncertainties. However, the drawbacks of this paper may come from the assumption. Using only common matrix B in assumption 1 seems conservative. Hence, this issue will be addressed in the future work. In addition, the signal dropout and trigger-event will be considered in near future as well.

\section{ACKNOWLEDGEMENTS}

This work belongs to the project with grant No: T2020-42TĐ/KHCN-GV funded by Ho Chi Minh City University of Technology and Education, Vietnam.

\section{REFERENCES}

[1] E. Sivaraman and S. Arulselvi, "Modeling of an inverted pendulum based on fuzzy clustering techniques, Expert Systems with Applications," Expert Systems with Applications, vol. 38, no. 11, pp. 13942-13949, 2011, doi: 10.1016/j.eswa.2011.04.201.

[2] M. Magdy, A. E. Marhomy, and M. A. Attia, "Modeling of inverted pendulum system with gravitational search algorithm optimized controller," Ain Shams Engineering Journal, vol. 10, no. 1, pp. 129-149, 2019, doi: 10.1016/j.asej.2018.11.001

[3] V. Kumar E and J. Jeromem, "Robust LQR Controller Design for Stabilizing and Trajectory Tracking of Inverted Pendulum," Procedia Engineering, vol. 64, pp. 169-178, 2013, doi: 10.1016/j.proeng.2013.09.088.

[4] A. Kharola, P. Patil, S. Raiwani, and D. Rajput, "A comparison study for control and stabilisation of inverted pendulum on inclined surface (IPIS) using PID and fuzzy controllers," Perspectives in Science, vol. 8, pp. 187-190, 2016, doi: 10.1016/j.pisc.2016.03.016.

[5] M. Bettayeb, C. Boussalem, R. Mansouri, and U. M. Al-Saggaf, "Stabilization of an inverted pendulum-cart system by fractional PI-state feedback," ISA Transactions, vol. 53, no. 2, pp. 508-516, 2014, doi: 10.1016/j.isatra.2013.11.014.

[6] E. Aranda-Escolástico, M. Guinaldo, F. Gordillo, and S. Dormido, "A novel approach to periodic event-triggered control: Design and application to the inverted pendulum," ISA Transactions, vol. 65, pp. 327-338, 2016, doi: 10.1016/j.isatra.2016.08.019.

[7] A. Ilyas Roose, S. Yahya, and H. Al-Rizzo, "Fuzzy-logic control of an inverted pendulum on a cart," Computers and Electrical Engineering, vol. 61, pp. 31-47, 2017, doi: 10.1016/j.compeleceng.2017.05.016.

[8] S. Chatterjee and S. K. Das, "An Analytical Formula for Optimal Tuning of the State Feedback Controller Gains for the State Feedback Controller Gains for the Cart-Inverted Pendulum System," IFAC PapersOnLine, vol. 51, no. 1, pp. 668-672, 2018, doi: 10.1016/j.ifacol.2018.05.112.

[9] X. J. Su, F. Q. Xia, J. X. Liu, and L. G. Wu, "Event-triggered fuzzy control of nonlinear systems with its application to inverted pendulum systems," Automatica, vol. 94, pp. 236-248, 2018, doi: 10.1016/j.automatica.2018.04.025.

[10] R. Bitirgenm, M. Hancer, and I. Bayezit, "All Stabilizing State Feedback Controller for Inverted Pendulum Mechanism," IFAC PapersOnLine, vol. 51, no. 4, pp. 346-351, 2018, doi: 10.1016/j.ifacol.2018.06.089.

[11] A. Chalanga, M. Patil, B. Bandyopadhyay, and H. Arya, "Output regulation using new sliding surface with an implementation on inverted pendulum system," European Journal of Control, vol. 45, pp. 85-91, 2019, doi: 10.1016/j.ejcon.2018.09.011.

[12] S. Irfan, A. Mehmood, M. T. Razzaq, and J. Iqbal, "Advanced sliding mode control techniques for Inverted Pendulum: Modelling and simulation," Engineering Science and Technology, vol. 21, pp. 753-759, 2018, doi: 10.1016/j.jestch.2018.06.010. 
[13] I. Jmel, H. Dimassi, S. Hadj-Said, and F. M. Sahli, "An adaptive sliding mode observer for inverted pendulum under mass variation and disturbances with experimental validation," ISA Transactions, vol. 102, pp. 264-279, 2020, doi: 10.1016/j.isatra.2020.02.029.

[14] F. Cacace, A. Germani, and C. Manes, "A New Approach to Design Interval Observers for Linear Systems," IEEE Transactions on Automatic Control, vol. 60, no. 6, pp. 1665-1670, 2015, doi: 10.1109/TAC.2014.2359714.

[15] V. P. Vu and W. J. Wang, "Observer design for a discrete time T-S fuzzy system with uncertainties," 2015 IEEE International Conference on Automation Science and Engineering (CASE), Gothenburg, Sweden, 2015, pp. 1262-1267, doi: 10.1109/CoASE.2015.7294272.

[16] V. P. Vu, W. J. Wang, J. M. Zurada, H. C. Chen, and C. H. Chiu, "Unknown input method based observer synthesis for a discrete time uncertain T-S fuzzy system," IEEE Transactions on Fuzzy Systems, vol. 26, no. 2, pp. 761-770, 2018, doi: 10.1109/TFUZZ.2017.2688388.

[17] D. Gutierrez, H. Rios, J.A. Rosales, and R. Galvan-Guerra, "Finite and Fixed-Time State Estimation: PendulumCart System," IFAC PapersOnLine, vol. 50, no. 1, pp. 7139-7144, 2017, doi: 10.1016/j.ifacol.2017.08.550.

[18] G. Pang, J. Cao, X. Y. Chen, and J. L. Qiu, "Simultaneous fault detection and antisaturated control based on dynamic observer for inverted pendulum control system," International Journal of Robust and Nonlinear Control, vol. 29, no. 1, pp. 3279-3295, 2019, doi: 10.1002/rnc.4550.

[19] S. Khatoon, D. K. Chaturvedi, N. Hasan, and M. Istiyaque, "Observer Based Controller Design for Inverted Pendulum System," 2018 2nd IEEE International Conference on Power Electronics, Intelligent Control and Energy Systems (ICPEICES), 2018, pp. 1046-1051, doi: 10.1109/ICPEICES.2018.8897379.

[20] S. Boyd, L. E. Ghaoui, E. Feron, and V. Balakrishnan, "Linear Matrix Inequalities in System and Control Theory," Society for Industrial and Applied Mathematics (SIAM), pp. 1-205, 1994.

[21] M. El-Bardini, and A. M. El-Nagar, "Interval type-2 fuzzy PID controller for uncertain nonlinear inverted pendulum system," ISA Transactions, vol. 53, no. 3, pp. 732-743, 2014, doi: 10.1016/j.isatra.2014.02.007.

[22] J. Lee, R. Mukherjee, and H. K. Khalil, "Output feedback stabilization of inverted pendulum on a cart in the presence of uncertainties," Automatica, vol. 54, pp. 146-157, 2015, doi: 10.1016/j.automatica.2015.01.013.

[23] J. L. Zhang, W. Zhang, "Adaptive Fuzzy Sliding Mode Control for Uncertain Inverted Pendulum System," Applied Mechanics and Materials, vol. 273, pp 683-688, 2013, doi: 10.4028/www.scientific.net/AMM.273.683.

[24] Y. F. Chen and A. C. Huang, "Adaptive control of rotary inverted pendulum system with time-varying uncertainties," Nonlinear Dynamics, vol. 76, pp. 95-102, 2014, doi: 10.1007/s11071-013-1112-4.

[25] C. Sarkar and A. Sengupta, "LMI based LSVF Control of a Class of Nonlinear Systems with Parametric Uncertainty: An Application to an Inverted Pendulum System," International Journal of Control, vol. 89, no. 12, pp. 2520-2532, 2016, doi: 10.1080/00207179.2016.1168528

[26] R. Kumari, J. Dey, S. K. Pandey, A. Chakraborty, and R. Mondal, " $\mu$-synthesis Controller for Robust Stabilization of Cart Inverted Pendulum System," 2017 4th IEEE Uttar Pradesh Section International Conference on Electrical, Computer and Electronics (UPCON), 2017, pp. 300-304, doi: 10.1109/UPCON.2017.8251064.

[27] A. K. Jayaprakash, K. B. Kidambi, W. MacKunis, S. V. Drakunov, and M. Reyhanoglu, "Finite-Time State Estimation for an Inverted Pendulum under Input-Multiplicative Uncertainty," Robotics, vol. 9, no. 4, pp. 1-26, 2020, Art. no. 87, doi: 10.3390/robotics 9040087

[28] S. H. Li, J. Yang, W. H. Chen, and X. S. Chen, "Disturbance Observer-based Control: Methods and Applications," Broken Sound Parkway NW: CRC Press Taylor and Francis Group, 2014.

[29] J. Han, H. G. Zhang, Y. C. Wang, and Y. Liu, "Disturbance observer-based fault estimation and dynamic output feedback fault tolerant control for fuzzy systems with local nonlinear model," ISA Transactions, vol. 59, pp. 114-124, 2015, doi: 10.1016/j.isatra.2015.08.015.

[30] T. Poloni, I. Kolmanovsky, and B. Rohal'-Ilkiv, "Input Disturbance Observer-Based Control: Case Studies," Journal of Dynamic Systems Measurement and Control, vol. 140, no. 1, pp. 1-8, 2018, doi: 10.1115/1.4037298.

[31] V. P. Vu and W. J. Wang, "State/Disturbance observer synthesis for T-S fuzzy system with the enlarge class of disturbances," IEEE Transactions on Fuzzy Systems, vol. 26, no. 6, pp. 3645-3659, 2018, doi: 10.1109/TFUZZ.2018.2841858.

[32] V. P. Vu and W. J. Wang, "Observer-based controller synthesis for uncertain polynomial systems," IET Control Theory and Applications, vol.12, no.1, pp. 29-37, 2018, doi: 10.1049/iet-cta.2017.0489.

[33] H. T. Hoang, "Intelligent Control System," published by Vietnam National University Press, 2006.

[34] J. S. Yeh, W. Chang, and W. J. Wang, "Unknown input based observer synthesis for uncertain T-S fuzzy systems," IET Control Theory and Applications, vol. 9, no. 5, pp. 729-735, 2015, doi: 10.1049/iet-cta.2014.0705.

[35] A. J. Laub, "Matrix Analysis for Scientists and Engineers," Philadelphia, PA, USA: SIAM, 2005. 